\title{
The problems of $n$-valence in some classes of analytic functions
}

\author{
Magdalena Sobczak-Kneć Katarzyna Trạbka-Więclaw*
}

\begin{abstract}
In this paper we determine some examples of the domains of $n$-valence for functions which are analytic with classical normalization and locally univalent. This problem has not been studied yet. It seems to be rather difficult. We formulate a few conjectures concerning some subclasses of typically real functions and odd typically real functions.
\end{abstract}

\section{Introduction}

Suppose that $\mathrm{T}$ denotes the well-known family of all typically real functions, i.e. functions which are analytic in the unit disc $\mathbb{U}=\{z \in \mathbb{C}:|z|<1\}$, normalized by $f(0)=f^{\prime}(0)-1=0$ and such that $\mathfrak{I m} z \mathfrak{I m} f(z) \geq 0$ for $z \in \mathbb{U}$. Moreover, let $\mathrm{T}^{(2)}$ be the class which consists of all odd typically real functions, that is, $\mathrm{T}^{(2)}=\{f \in \mathrm{T}: f(z)=-f(-z), z \in \mathbb{U}\}$.

The univalence problems in the class of typically real functions were considered by Golusin in [1]. He proved that the domain of local and global univalence for typically real functions coincide. The problems of univalence for the class of odd typically real functions were investigated by Koczan and Zaprawa in [4] and [5]. They proved that there can be infinitely many domains of univalence for odd typically real functions and they determined some of these domains.

\footnotetext{
${ }^{*}$ Corresponding author

Received by the editors in July 2013.

Communicated by H. De Schepper.

2010 Mathematics Subject Classification : Primary 30C45; Secondary 30C55.

Key words and phrases : Radius of $n$-valence, domain of $n$-valence, typically real functions, odd typically real functions, locally univalent functions.
} 
In this paper we study the problems of $n$-valence in some classes of analytic functions. This problem has not been studied yet. It seems to be rather difficult. Recall now the definition of $n$-valent function (see e.g. [2], vol. I, p.89).

Definition 1. A function $f$ analytic in a domain $D$ is said to be n-valent in $D$, if for each $w_{0}$ the equation $f(z)=w_{0}$ has at most $n$ roots in $D$ (where the roots are counted in accordance with their multiplicity) and if there is some $w_{1}$ such that the equation $f(z)=w_{1}$ has exactly $n$ roots in $D$.

Now we formulate a few definitions connected with $n$-valence problems. Let $\mathcal{A}$ be the class of all analytic functions. A domain $D \subset \mathbb{U}$ is called the domain of $n$-valence of the class $\mathrm{A} \subset \mathcal{A}$, if every function from the class $\mathrm{A}$ is at most $n$-valent in $D$ and for every domain $\Omega$ such that $D \varsubsetneqq \Omega \subset \mathbb{U}$ there exists a function which is at least $(n+1)$-valent in $\Omega$. A domain $D \subset \mathbb{U}$ is called the domain of $n$-valence of the function $f \in \mathcal{A}$, if the function $f$ is $n$-valent in $D$ and for every domain $\Omega$ such that $D \varsubsetneqq \Omega \subset \mathbb{U}$ the function $f$ is at least $(n+1)$-valent in $\Omega$. We say that $r_{n}(\mathrm{~A})$ is the radius of $n$-valence of the class $\mathrm{A} \subset \mathcal{A}$, if all functions of the class $\mathrm{A}$ are at most $n$-valent in the disc $|z|<r_{n}(\mathrm{~A})$ and for every $r \in\left(r_{n}(\mathrm{~A}), 1\right)$ there exists a function in A which is at least $(n+1)$-valent in the disc $|z|<r$. We say that $r_{n}$ is the radius of n-valence of the function $f \in \mathcal{A}$, if the function $f$ is $n$-valent in the disc $|z|<r_{n}$ and the function $f$ is at least $(n+1)$-valent in every disc $|z|<r$, $r \in\left(r_{n}, 1\right)$.

\section{Main results}

Let us denote by $F$ a typically real function, called the universal function, which was introduced by Goodman in the paper [3], that is,

$$
F(z)=\frac{1}{\pi} \tan \frac{\pi z}{1+z^{2}}, \quad z \in \mathbb{U} .
$$

We determine the radius of $n$-valence of the function $F$. We know from [3] that $F \in \mathrm{T}$ and $F^{\prime}(z) \neq 0, z \in \mathbb{U}$. This means that the function $F$ is typically real and locally univalent. One can observe that

$$
F(z)=\frac{1}{\pi i} \frac{1-\exp \left\{\frac{-2 i \pi z}{1+z^{2}}\right\}}{1+\exp \left\{\frac{-2 i \pi z}{1+z^{2}}\right\}}=\frac{1}{\pi i} \frac{1-\mathrm{e}^{-i \omega}}{1+\mathrm{e}^{-i \omega}} \quad \text { for } \quad \omega(z)=\frac{2 \pi z}{1+z^{2}}, \quad z \in \mathbb{U} .
$$

The function $\omega \mapsto \mathrm{e}^{i \omega}$ is $n$-valent function in every curvelinear strip with the width in the direction to the real axis equals to $2 n \pi$, i.e. the intersection of this strip with any straight line parallel to the real axis is the segment of the measure $2 n \pi$. Hence let us consider vertical strip

$$
\{\omega \in \mathbb{C}:|\mathfrak{R e} \omega|<n \pi\}, \quad n \in \mathbb{N} .
$$

Thus for each $n \in \mathbb{N}$ we determine the subset of $\mathbb{U}$ described by the inequality

$$
\left|\mathfrak{R e} \frac{2 \pi z}{1+z^{2}}\right|<n \pi
$$


that is, by the inequality

$$
\left|\mathfrak{R e} \frac{z}{1+z^{2}}\right|<\frac{n}{2}
$$

Let $z=r \mathrm{e}^{i \theta}, \theta \in[0, \pi / 2]$. Then

$$
\mathfrak{R e} \frac{z}{1+z^{2}}=\frac{r\left(1+r^{2}\right) \cos \theta}{r^{4}+2 r^{2} \cos 2 \theta+1}=\frac{r\left(1+r^{2}\right) \cos \theta}{r^{4}+4 r^{2} \cos ^{2} \theta+1-2 r^{2}} .
$$

From this and (2) we obtain the following inequality

$$
\frac{r\left(1+r^{2}\right) \cos \theta}{r^{4}+4 r^{2} \cos ^{2} \theta+1-2 r^{2}}<\frac{n}{2}
$$

or equivalently, putting $\cos \theta=t,|t| \leq 1$, we get

$$
\frac{r\left(1+r^{2}\right) t}{r^{4}+4 r^{2} t^{2}+1-2 r^{2}}<\frac{n}{2}
$$

We find the largest $r$ which satisfies the inequality above for every $t \in[-1,1]$. Define

$$
w(t)=4 r^{2} t^{2}-\frac{2}{n} r\left(1+r^{2}\right) t+\left(r^{2}-1\right)^{2} .
$$

We solve the following inequality

$$
\min _{t \in[-1,1]} w(t)>0
$$

We obtain

$$
\begin{aligned}
\min _{t \in[-1,1]} w(t) & = \begin{cases}w\left(t_{v}\right) & \text { for } t_{v} \leq 1, \\
w(1) \quad \text { for } \quad t_{v}>1,\end{cases} \\
& = \begin{cases}\left(r^{2}-1-\frac{1+r^{2}}{2 n}\right)\left(r^{2}-1+\frac{1+r^{2}}{2 n}\right) & \text { for } 2 n-\sqrt{4 n^{2}-1} \leq r \leq 1, \\
\left(r^{2}+1\right)\left(r^{2}+1-\frac{n r}{2}\right) & \text { for } 0 \leq r<2 n-\sqrt{4 n^{2}-1},\end{cases}
\end{aligned}
$$

where $t_{v}=\frac{1+r^{2}}{4 r n}$ is the vertex of the parabola. It is easy to check that $w(1)>0$ for $0 \leq r<2 n-\sqrt{4 n^{2}-1}$. Thus from $w\left(t_{v}\right)>0$ we have

$$
\left\{\begin{array}{l}
\left(r^{2}-1-\frac{1+r^{2}}{2 n}\right)\left(r^{2}-1+\frac{1+r^{2}}{2 n}\right)>0 \\
2 n-\sqrt{4 n^{2}-1} \leq r \leq 1
\end{array}\right.
$$

or equivalently

$$
\left\{\begin{array}{l}
r^{2}<\frac{2 n-1}{2 n+1} \\
2 n-\sqrt{4 n^{2}-1} \leq r \leq 1
\end{array} \Leftrightarrow 2 n-\sqrt{4 n^{2}-1} \leq r<\sqrt{\frac{2 n-1}{2 n+1}} .\right.
$$

Therefore from the inequality (3) we get $0 \leq r<\sqrt{\frac{2 n-1}{2 n+1}}$. Hence we have proved the following theorem. 
Theorem 1. The function $F$ given by (1) is n-valent function in the disc $|z|<\sqrt{\frac{2 n-1}{2 n+1}}$.

Corrolary 1. The radius of univalence of the function $F$ given by (1) is equal to $\sqrt{3} / 3$.

The domains of $n$-valence of a given function could not be unique. There exist functions having infinitely many domains of univalence (see for example [4]). We show that the universal function $F$ has infinitely many domains of $n$-valence and we determine three of them.

Let us consider a strip bounded by two straight lines $l_{1}: \omega=-n \pi+y e^{i \varphi}$ and $l_{2}: \omega=n \pi+y e^{i \varphi}, n \in \mathbb{N}, \varphi \in(0, \pi)$ (the strip includes 0 ). Obviously this strip has the width in the direction to the real axis equals to $2 n \pi$, i.e. the intersection of this strip with any straight line parallel to the real axis is the segment of the measure $2 n \pi$. So the inverse images of the strip through the function $z \mapsto \frac{\pi z}{1+z^{2}}$ are the domains of $n$-valence of the function $F$ given by (1). Thus we get the oneparameter family of domains of $n$-valence of the function $F$; there are infinitely many such domains.

Assume that $E_{n}$ is a set which is bounded, symmetric with the respect to both axes of the complex plane and which has the boundary in the first quadrant of the coordinate system given by the polar equation $\rho=r(\theta) \mathrm{e}^{i \theta}$, where

$$
r(\theta)= \begin{cases}1 & \text { for } \theta \in\left[0, \arccos \frac{1}{n}\right), \\ \frac{1}{2 n}\left(\alpha-\sqrt{\alpha^{2}-4 n^{2}}\right) & \text { for } \theta \in\left[\arccos \frac{1}{n}, \frac{\pi}{2}\right],\end{cases}
$$

where $\alpha=\cos \theta+\sqrt{\cos ^{2} \theta+4 n^{2} \sin ^{2} \theta}$. In Figures 1 and 2 we can see the sets $E_{1}$, $E_{5}$ and the discs of univalence $|z|<\sqrt{3} / 3$ and of 5 -valence $|z|<3 \sqrt{11} / 11$ of the function $F$.

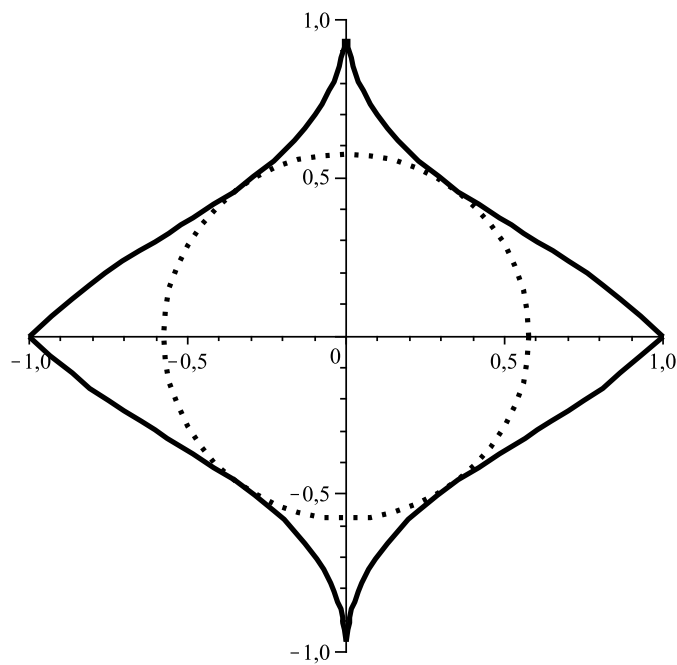

Figure 1: The boundary of the set $E_{1}$ (solid line) and the disc of univalence $|z|<\sqrt{3} / 3$ (dot line).



Figure 2: The boundary of the set $E_{5}$ (solid line) and the disc of 5-valence $|z|<3 \sqrt{11} / 11$ (dot line). 
Theorem 2. The set $E_{n}$ is the domain of n-valence of the function $F$ given by (1).

Proof. The universal function $F$ is $n$-valent when

$$
-n \pi<\mathfrak{I m} \frac{2 \pi i z}{1+z^{2}}<n \pi, \quad \text { that is, when } \quad-n \pi<\mathfrak{R e} \frac{2 \pi z}{1+z^{2}}<n \pi
$$

Thus to determine the domain of $n$-valence, it is sufficient to solve the following equation

$$
\mathfrak{R e} \frac{z}{1+z^{2}}=\frac{n}{2}
$$

Let $z=r \mathrm{e}^{i \theta}, \theta \in[0, \pi / 2]$. Therefore the equation above takes the form

$\frac{r\left(1+r^{2}\right) \cos \theta}{r^{4}+4 r^{2} \cos ^{2} \theta+1-2 r^{2}}=\frac{n}{2} \quad \Leftrightarrow \quad r^{4}-\frac{2}{n} r\left(r^{2}+1\right) \cos \theta+4 r^{2} \cos ^{2} \theta-2 r^{2}+1=0$.

Putting $r+\frac{1}{r}=x, x \geq 2$, we obtain the following equation

$$
x^{2}-\frac{2}{n} x \cos \theta-4 \sin ^{2} \theta=0,
$$

which has two solutions of the form:

$$
\begin{aligned}
& x_{1}=\frac{1}{n}\left(\cos \theta+\sqrt{\cos ^{2} \theta+4 n^{2} \sin ^{2} \theta}\right), \\
& x_{2}=\frac{1}{n}\left(\cos \theta-\sqrt{\cos ^{2} \theta+4 n^{2} \sin ^{2} \theta}\right) .
\end{aligned}
$$

Notice that $x_{2} \leq 0$. The condition $x_{1} \geq 2$ implies

$$
\begin{gathered}
\frac{1}{n}\left(\cos \theta+\sqrt{\cos ^{2} \theta+4 n^{2} \sin ^{2} \theta}\right) \geq 2 \Leftrightarrow \cos \theta\left(\cos \theta-\frac{1}{n}\right) \leq 0 \\
\Leftrightarrow \quad 0 \leq \cos \theta \leq \frac{1}{n} \Leftrightarrow \theta \in\left[\arccos \frac{1}{n}, \frac{\pi}{2}\right] .
\end{gathered}
$$

Hence $\theta \in\left[\arccos \frac{1}{n}, \frac{\pi}{2}\right]$ and $r(\theta)$ is the solution of the equation

$$
r^{2}-\frac{r}{n}\left(\cos \theta+\sqrt{\cos ^{2} \theta+4 n^{2} \sin ^{2} \theta}\right)+1=0
$$

which belongs to the interval $[0,1]$, that is,

$$
\begin{aligned}
r(\theta) & =\frac{1}{2 n}\left(\cos \theta+\sqrt{\cos ^{2} \theta+4 n^{2} \sin ^{2} \theta}\right)+ \\
& -\frac{1}{2 n} \sqrt{\left(\cos \theta+\sqrt{\cos ^{2} \theta+4 n^{2} \sin ^{2} \theta}\right)^{2}-4 n^{2}} .
\end{aligned}
$$

Therefore, the proof is complete. 
Suppose that $B_{n}$ is a set which is bounded, symmetric with the respect of the real axis and which the boundary coincides with the segment $[-i, i]$ and the arc given by the polar equation $\rho=r(\theta) \mathrm{e}^{i \theta}$, where

$$
r(\theta)= \begin{cases}1 & \text { for } \theta \in\left(-\arccos \frac{1}{2 n}, \arccos \frac{1}{2 n}\right), \\ \frac{1}{4 n}\left(\beta-\sqrt{\beta^{2}-16 n^{2}}\right) & \text { for } \quad \theta \in\left[-\frac{\pi}{2},-\arccos \frac{1}{2 n}\right] \cup\left[\arccos \frac{1}{2 n}, \frac{\pi}{2}\right],\end{cases}
$$

where $\beta=\cos \theta+\sqrt{\cos ^{2} \theta+16 n^{2} \sin ^{2} \theta}$. Moreover, let $\widetilde{B}_{n}$ denote set which is symmetric to the set $B_{n}$ with the respect of the imaginary axis. In Figure 3 we can see the sets $B_{1}$ and $\widetilde{B}_{1}$.
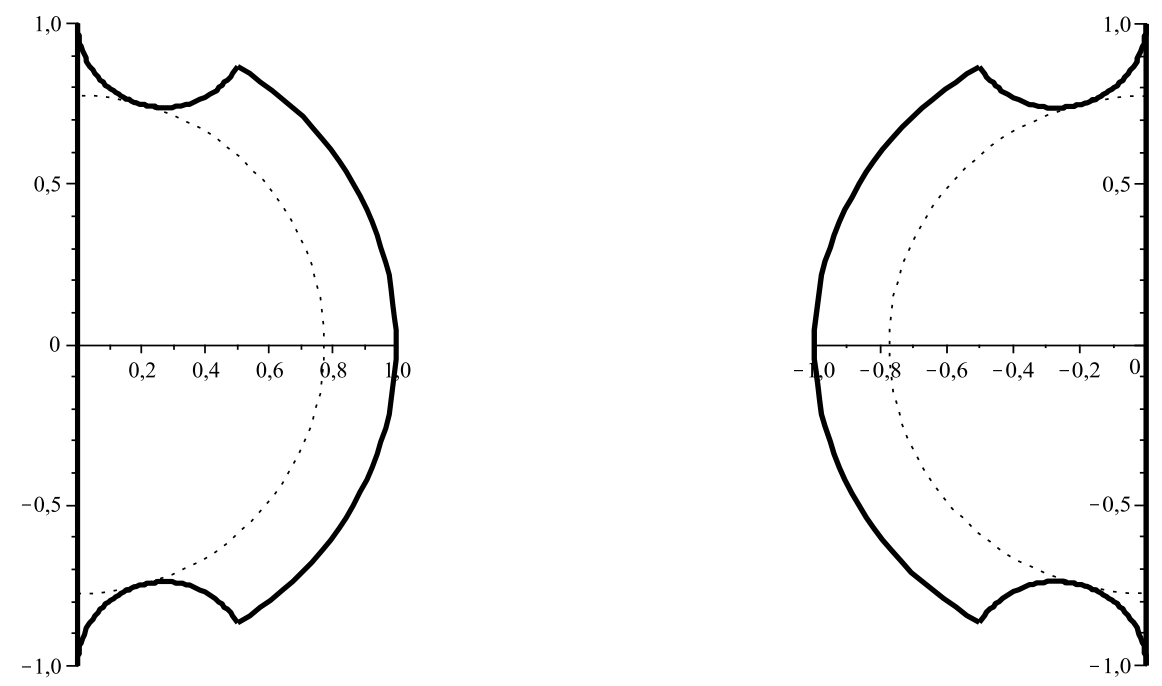

Figure 3: The boundary of the set $B_{1}$ and of the set $\widetilde{B}_{1}$.

Theorem 3. The sets $B_{n}$ and $\widetilde{B}_{n}$ are the domain of n-valence of the function $F$ given by (1).

The proof is similar to the proof of Theorem 2, but we consider vertical strip

$$
\{\omega \in \mathbb{C}: 0<\mathfrak{R e} \omega<2 n \pi\}, \quad n \in \mathbb{N}
$$

and we solve the following equation

$$
\mathfrak{R e} \frac{z}{1+z^{2}}=n \text {. }
$$

In each domain of $n$-valence of a given function, there exist domains of $n$-valence of a given class. We know that all functions from a given class $\mathrm{A} \subset \mathcal{A}$ are $n$-valent in the disc $|z|<r_{n}(\mathrm{~A})$. It may suggest that the intersection of the domains of $n$-valence of a given class $\mathrm{A}$ includes the disc $|z|<r_{n}(\mathrm{~A})$. Nevertheless, let us notice that in the class $\{F\}$ (i.e. the class which consists of one function $F$ ) the intersection of the domains of $n$-valence is included in the intersection of the sets $B_{n}$ and $\widetilde{B}_{n}$, that is, in the set $B_{n} \cap \widetilde{B}_{n}$, which is the empty set. Thus there 
exist classes for which the intersection of the domains of $n$-valence is the empty set.

And finally, we formulate the following conjectures. Let $\mathcal{A}_{L U}$ be the subclass of $\mathcal{A}$ which consists of functions with classical normalization locally univalent in $\mathbb{U}$, i.e.

$$
\mathcal{A}_{L U}=\left\{f \in \mathcal{A}: f(z)=z+a_{2} z^{2}+\ldots, f^{\prime}(z) \neq 0, z \in \mathbb{U}\right\} .
$$

\section{Conjecture 1.}

(i) The radius of $n$-valence of the function $F$ given by (1) is the radius of $n$-valence of the class $\mathrm{T}^{(2)} \cap \mathcal{A}_{L U}$ and of the class $\mathrm{T} \cap \mathcal{A}_{L U}$.

(ii) The domains of n-valence of the function $F$ given by (1) are the domains of n-valence of the class $\mathrm{T}^{(2)} \cap \mathcal{A}_{L U}$ and of the class $\mathrm{T} \cap \mathcal{A}_{L U}$.

\section{Some examples}

Similar problems one can consider in the classes generated by functions which are not locally univalent. First, let us introduce some notation: $\mathbb{U}(p, r)=$ $\{z \in \mathbb{C}:|z-p|<r\}$ (so $\mathbb{U}(0,1)=\mathbb{U}), \mathbb{U}^{+}=\{z \in \mathbb{U}: \mathfrak{I m} z>0\}, \mathbb{U}^{-}=$ $\{z \in \mathbb{U}: \mathfrak{I m} z<0\}, \mathbb{C}^{+}=\{z \in \mathbb{C}: \mathfrak{I m} z>0\}$ and for a set A let $\overline{\mathrm{A}}$ be a closure of a set $\mathrm{A}$.

Now let us consider the function

$$
h(z)=z+z^{2} .
$$

Notice that the function $h$ is 2-valent but it is not neither typically-real nor locally univalent. Let us observe that $h\left(\mathbb{U}^{+}\right)$is the set which is bounded by the curve $\Gamma_{1} \cup \Gamma_{2}$, where

$$
\begin{aligned}
& \Gamma_{1}:\left\{\begin{array}{l}
x(\theta)=\cos \theta+\cos 2 \theta \\
y(\theta)=\sin \theta+\sin 2 \theta
\end{array}, \theta \in[0, \pi]\right. \\
& \Gamma_{2}=[0,2] .
\end{aligned}
$$

Since the function $h$ has real coefficients, so $h\left(\mathbb{U}^{-}\right)$is the set which is symmetric to $h\left(\mathbb{U}^{+}\right)$with the respect to the real axis of the complex plane. Thus, the set $h(\mathbb{U})$ can be presented as the union of two disjoint sets $G_{1}$ and $G_{2}$ such that all points of the set $G_{1}$ are taken by the function $h$ only once, and all points of the set $G_{2}$ are taken by $h$ twice. Hence, the set $G_{2}$ is the interior of the curve $\left\{h\left(e^{i \theta}\right): \theta \in[2 \pi / 3,4 \pi / 3]\right\}$. In Figure 5 we have the set $h(\mathbb{U})$.

Suppose that $D_{1}=\mathbb{U} \backslash\left(\overline{\mathbb{U}(-1,1)} \cap \overline{\mathbb{C}^{+}}\right), D_{2}$ is the set which is symmetric to $D_{1}$ with respect to the real axis of the complex plane and $D_{3}=\mathbb{U} \cap$ $\{z \in \mathbb{C}: \mathfrak{R e} z>-1 / 2\}$. In Figure 4 we can see the sets $D_{1}, D_{2}$ and $D_{3}$.

Theorem 4. The sets $D_{1}, D_{2}, D_{3}$ are the domains of univalence of the function $h(z)=$ $z+z^{2}$. 
Proof. The function $h$ is univalent in $\mathbb{U}^{-}$, because for all $z_{1}, z_{2} \in \mathbb{U}^{-}$we have $h\left(z_{1}\right)=h\left(z_{2}\right) \Leftrightarrow z_{1}+z_{1}^{2}=z_{2}+z_{2}^{2}$. Therefore, $z_{1}=z_{2}$ or $z_{1}+z_{2}=1$. The second equality is not true for $z_{1}, z_{2} \in \mathbb{U}^{-}$. Thus, $h$ is univalent also in $\mathbb{U}^{+}$and in each subset of $\mathbb{U}^{+}$.

First we prove that $D_{1}$ is the domain of univalence of the function $h$. Denote by $E$ the subset of $\mathbb{U}^{+}$such that $h(E)=h(\mathbb{U}) \backslash \overline{h\left(\mathbb{U}^{-}\right)}$. To this end, we solve the following equation

$$
\begin{gathered}
h(z)=h\left(e^{i \theta}\right), \theta \in[2 \pi / 3, \pi] \\
z^{2}+z=e^{2 i \theta}+e^{i \theta} \Leftrightarrow\left(z-e^{i \theta}\right)\left(z+e^{i \theta}+1\right)=0 \Leftrightarrow z=e^{i \theta} \vee z=-e^{-i \theta}-1 .
\end{gathered}
$$

Since

$$
\begin{gathered}
\left\{h\left(e^{i \theta}\right): \theta \in(0,2 \pi / 3)\right\}=\partial h(\mathbb{U}) \cap \mathbb{C}^{+} \\
\left\{h\left(-e^{-i \theta}-1\right): \theta \in(2 \pi / 3, \pi)\right\}=\partial h\left(\mathbb{U}^{-}\right) \cap \mathbb{C}^{+}
\end{gathered}
$$

and

$$
\{h(x): x \in[0,1)\}=[0,2),
$$

then $h(E)=G_{1} \cap \mathbb{C}^{+}$. Hence, $h(E) \cap h\left(\mathbb{U}^{-}\right)=\varnothing$ and $h(E) \cup h\left(\mathbb{U}^{-}\right) \cup[0,2)=$ $h(\mathbb{U})$.

Analogously, we can prove that $D_{2}$ is the domain of univalence of the function $h$.

Now we prove that $D_{3}$ is the domain of univalence of the function $h$. Because the function $h$ is univalent in $\mathbb{U}^{+}$, so it is univalent also in $H^{+}=D_{3} \cap \mathbb{C}^{+}$. Since we have:

$$
\begin{gathered}
\left\{h\left(e^{i \theta}\right): \theta \in(0,2 \pi / 3)\right\}=\partial h(\mathbb{U}) \cap \mathbb{C}^{+}, \\
\{h(i t-1 / 2): t \in(0, \sqrt{3} / 2)\}=(-1,-1 / 4), \\
\{h(x): x \in(-1 / 2,1)\}=(-1 / 4,2)
\end{gathered}
$$

and the function $h$ is locally univalent for $z \neq-1 / 2$, thus $h\left(H^{+}\right)=h(\mathbb{U}) \cap \mathbb{C}^{+}$.

Analogously, the function $h$ is univalent in $H^{-}=D_{3} \cap \mathbb{C}^{+}$and $h\left(H^{-}\right)=$ $h(\mathbb{U}) \cap \mathbb{C}^{-}$.
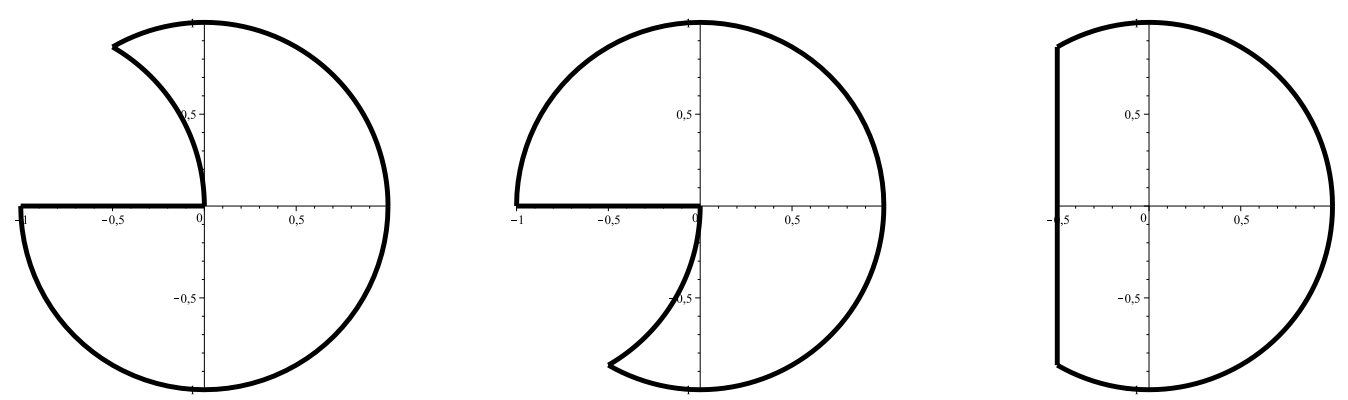

Figure 4: The boundaries of the sets $D_{1}, D_{2}$ and $D_{3}$. 


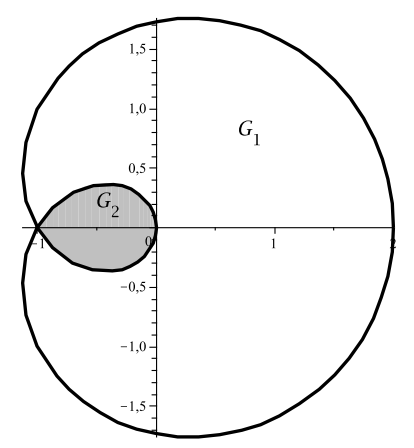

Figure 5: The boundary of the set $h(\mathbb{U})$.

Finally, we present (without proof) one more example of the function with their domains of univalence. Namely, the function

$$
g(z)=z+z^{2}+\frac{1}{3} z^{3}
$$

which is locally univalent but it is not typically-real. Let $H_{1}=\mathbb{U} \backslash \overline{\mathbb{U}\left(-\frac{3}{2}+\frac{\sqrt{3}}{2} i, 1\right)}$, $H_{2}=\mathbb{U} \backslash \overline{\mathbb{U}\left(-\frac{3}{2}-\frac{\sqrt{3}}{2} i, 1\right)}$ and $H_{3}=\mathbb{U} \cap\left\{z:|\arg (z+1)|<\frac{\pi}{3}\right\}$. In Figure 6 we can see the sets $H_{1}, H_{2}, H_{3}$ and in Figure 7 - the set $g(\mathbb{U})$.


Figure 6: The boundaries of the sets $H_{1}, H_{2}$ and $H_{3}$.



Figure 7: The boundary of the set $g(\mathbb{U})$. 
Theorem 5. The sets $H_{1}, H_{2}, H_{3}$ are the domains of univalence of the function $g(z)=$ $z+z^{2}+\frac{1}{3} z^{3}$.

\section{References}

[1] Golusin G. On typically real functions. Mat. Sb. 1950; 27(69): 201-218.

[2] Goodman AW. Univalent functions. Tampa, Florida, USA: Mariner Publishing Company Inc. 1983; Vol. I: 89.

[3] Goodman AW. The domain covered by a typically real function. Proc. Amer. Math. Soc. 1977; 64: 233-237.

[4] Koczan L, Zaprawa P. Domains of univalence for typically real odd functions. Complex Variables 2003; 48 No. 1: 1-17.

[5] Koczan L, Zaprawa P. On domains of univalence for the class of typically real functions. Folia Sci. Univ. Tech. Resoviensis 2001; Math. 25 (190): 81-89.

Department of Mathematics,

Mechanical Engineering Faculty,

Lublin University of Technology,

Lublin, Poland

email:m.sobczak-knec@pollub.pl,k.trabka@pollub.pl 